\title{
Uncoupling of glycolysis from glucose oxidation accompanies the development of heart failure with preserved ejection fraction
}

\author{
Natasha Fillmore, Jody L. Levasseur, Arata Fukushima, Cory S. Wagg, Wei Wang, Jason R. B. Dyck
} and Gary D. Lopaschuk*

\begin{abstract}
Background: Alterations in cardiac energy metabolism contribute to the development and severity of heart failure (HF). In severe HF, overall mitochondrial oxidative metabolism is significantly decreased resulting in a reduced energy reserve. However, despite the high prevalence of HF with preserved ejection fraction (HFpEF) in our society, it is not clear what changes in cardiac energy metabolism occur in $\mathrm{HFpEF}$, and whether alterations in energy metabolism contribute to the development of contractile dysfunction.
\end{abstract}

Methods: We directly assessed overall energy metabolism during the development of HFpEF in Dahl salt-sensitive rats fed a high salt diet (HSD) for 3, 6 and 9 weeks.

Results: Over the course of 9 weeks, the HSD caused a progressive decrease in diastolic function (assessed by echocardiography assessment of $E^{\prime} / A^{\prime}$ ). This was accompanied by a progressive increase in cardiac glycolysis rates (assessed in isolated working hearts obtained at 3, 6, and 9 weeks of HSD). In contrast, the subsequent oxidation of pyruvate from glycolysis (glucose oxidation) was not altered, resulting in an uncoupling of glucose metabolism and a significant increase in proton production. Increased glucose transporter (GLUT)1 expression accompanied this elevation in glycolysis. Decreases in cardiac fatty acid oxidation and overall adenosine triphosphate (ATP) production rates were not observed in early HF, but both significantly decreased as HF progressed to HF with reduced EF (i.e. 9 weeks of HSD).

Conclusions: Overall, we show that increased glycolysis is the earliest energy metabolic change that occurs during HFpEF development. The resultant increased proton production from uncoupling of glycolysis and glucose oxidation may contribute to the development of HFpEF.

Keywords: Mitochondria, Fatty acid oxidation, Energy metabolism, Diastolic dysfunction, Cardiac hypertrophy

\section{Background}

An abundance of evidence indicates that alterations in energy metabolism contribute to the severity of heart failure (Kato et al. 2010; Degens et al. 2006; Lei et al. 2004; Conway et al. 1991; Nascimben et al. 1995; Tian et al. 1996; Beer et al. 2002; Neubauer et al. 1999; Mori et al. 2013). This includes a decrease in overall cardiac mitochondrial oxidative metabolism as the severity of

\footnotetext{
* Correspondence: glopasch@ualberta.ca

Cardiovascular Research Centre, Mazankowski Alberta Heart Institute University of Alberta, Edmonton, Canada
}

heart failure increases (Conway et al. 1991; Nascimben et al. 1995; Tian et al. 1996; Beer et al. 2002; Neubauer et al. 1999; Lopaschuk et al. 2010). Improving cardiac efficiency, such as by either stimulating glucose oxidation or inhibiting fatty acid oxidation, can help to lessen the impact of this decrease in mitochondrial oxidative capacity, and can improve cardiac function in the failing heart (Kato et al. 2010; Masoud et al. 2014; Yamashita et al. 2009; Ussher et al. 2012; Stanley et al. 2005; Lopaschuk et al. 2003; Dyck et al. 2006; Dyck et al. 2004). However, there is not a consensus as to the importance of the specific energy metabolic changes to 
the development of heart failure. While it is generally believed that there is an increase in overall glucose metabolism in the failing heart, this may actually be specific to an increase in glucose uptake and glycolysis (Lei et al. 2004; Lopaschuk et al. 2010; Masoud et al. 2014). Whether mitochondrial oxidation of glucose, which supplies the majority of adenosine triphosphate (ATP) derived from glucose, is also increased in heart failure is debatable. In fact, myocardial glucose oxidation rates are decreased in mouse hearts subjected to cardiac hypertrophy and heart failure (Mori et al. 2013; Zhabyeyev et al. 2013; Zhang et al. 2013), and in pig hearts subjected to rapid-pacing induced heart failure (Schroeder et al. 2013).

The specific changes in glycolysis rates and glucose oxidation rates in heart failure are important because uncoupling of glycolysis and glucose oxidation has been shown to impair cardiac function. A selective increase in glycolysis relative to glucose oxidation uncouples glycolysis from glucose oxidation, which can result in the production of lactate and protons (Liu et al. 1996; Liu et al. 2002; Folmes et al. 2006). This rise in protons and drop in $\mathrm{pH}$ can reduce contractility of the adult heart by impairing troponin I sensitivity to calcium and inhibiting the slow calcium current (Chesnais et al. 1975; Vogel and Sperelakis 1977; Steenbergen et al. 1977; Schiaffino et al. 1993; Morimoto and Goto 2000). In addition, ATP is utilized to both remove these protons and maintain sodium and calcium homeostasis which decreases cardiac efficiency and contributes to the decrease in cardiac function (Lopaschuk et al. 2010).

Uncoupling of glycolysis and glucose oxidation may also contribute to the development of heart failure by increasing cardiac hypertrophy. Uncoupling of glycolysis and glucose oxidation is present in proliferative cells and is believed to be important in promoting cell growth. Otto Warburg first reported that cancer cells, which are characterized by high rates of proliferation, have high glycolysis rates even under aerobic conditions, a phenomenon called the "Warburg" effect (Vander Heiden et al. 2009; Warburg 1956). In addition, glycolysis is elevated in another form of cell growth, cardiac hypertrophy, which can lead to heart failure (Piao et al. 2010; Piao et al. 2013; Allard et al. 1994; Leong et al. 2002). A similar "Warburg" phenomena may also exist in the failing heart, which is frequently characterized by a relative rise in glycolysis and an overall decrease in mitochondrial oxidative metabolism (Lei et al. 2004; Conway et al. 1991; Nascimben et al. 1995; Tian et al. 1996; Beer et al. 2002; Neubauer et al. 1999; Lopaschuk et al. 2010; Zhang et al. 2013). This suggests that the coupling of glycolysis and glucose oxidation may be a promising target for the treatment of diseases characterized by abnormal cell growth. In fact, stimulation of glucose oxidation (by inhibition of pyruvate dehydrogenase kinase, PDK) has been reported to be beneficial in multiple scenarios, including treatment of cancer, T-cells, cardiac hypertrophy, and heart failure (Kato et al. 2010; Liu et al. 1996; Bonnet et al. 2007; Gerriets et al. 2015).

While there is a substantial amount of evidence to indicate that heart failure with reduced ejection fraction (HFrEF) is commonly characterized by an overall decrease in oxidative metabolism and relative increase in glycolysis (which can result in increased uncoupling of glycolysis and glucose oxidation), there is a scarcity of research on metabolism in another common form of heart failure, heart failure with preserved ejection fraction (HFpEF). In this study we therefore examined mitochondrial oxidative metabolism and glycolysis during the development of HFpEF. This was examined using the Dahl salt-sensitive rat, a well characterized model of HFpEF (Horgan et al. 2014; Rapp and Dene 1985; Klotz et al. 2006), and cardiac energy metabolism was assessed after 3 weeks, 6 weeks, or 9 weeks on a high salt diet (HSD).

\section{Methods \\ Animal protocol}

Eight week old male Dahl salt-sensitive rats were either fed a standard low salt diet containing $0.3 \% \mathrm{NaCl}$ (Research Diets, D10012G) or a high salt diet (HSD) (Research Diets, D11021901) containing $8 \% \mathrm{NaCl}$ to induce HFpEF. Control rats were kept on the low salt diet while treatment groups were fed the HSD for 3,6 , or 9 weeks. Food and water were provided ad libitum. Rats were kept on a $12 \mathrm{~h}$ light:12 h dark cycle. All procedures on animals were approved by the University of Alberta Health Sciences Animal Welfare Committee and conformed to the Canadian Council on Animal Care guidelines (Canadian Council on Animal Care 2017).

\section{Echocardiography}

In vivo cardiac function was assessed in rats anesthetized with $1-1.5 \%$ isoflurane using a Vevo 770 high resolution echocardiography imaging system (VisualSonics, Toronto) with a 30-MHz transducer (Zhong et al. 2010). Doppler and tissue doppler imaging were used to assess diastolic function: E'/A, E, E/A, E'/E, and isovolumetric relaxation time (IVRT). M-mode images were used to measure \% Ejection fraction (\%EF) and \% Fractional shortening (\%FS), to make left ventricle (LV) wall measurements [Interventricular septum end diastole (IVSd), LV internal diameter end diastole (LVIDd), LV posterior wall thickness end diastole (LVPWd), Interventricular septum end systole (IVSs), LV internal diameter end systole (LVIDs), LV posterior wall thickness end systole (LVPWs)], and to measure LV diameter and volume [left ventricular end diastolic diameter, left ventricular end systolic diameter, LV volume end diastole (LV Vol;d), LV volume end systole (LV Vol;s), and corrected LV mass]. 


\section{Isolated working heart perfusions}

Rats were anesthetized with sodium pentobarbital $(1 \mathrm{~g} / \mathrm{kg}$ $\mathrm{BW})$. Hearts were quickly excised from fully anesthetized rats, and were perfused in the working mode at a $11.5 \mathrm{mmHg}$ left atrial preload and $80 \mathrm{mmHg}$ aortic afterload, as previously described (Liu et al. 1996; Liu et al. 2002). Isolated working hearts were perfused with modified Krebs-Henseleit solution $(118.5 \mathrm{mM} \mathrm{NaCl}, 25 \mathrm{mM}$ $\mathrm{NaHCO}_{3}, 4.7 \mathrm{mM} \mathrm{KCl}, 1.2 \mathrm{mM} \mathrm{MgSO}_{4}, 1.2 \mathrm{mM} \mathrm{KH}_{2} \mathrm{PO}_{4}$, $2.5 \mathrm{mM} \mathrm{CaCl}_{2}$ ) supplemented with $5 \mathrm{mM}$ glucose, $0.5 \mathrm{mM}$ lactate, and $0.8 \mathrm{mM}$ palmitate bound to $3 \%$ fatty acid-free bovine serum albumin (BSA). To measure palmitate oxidation, glucose oxidation, glycolysis, and lactate oxidation $\left[9,10-{ }^{3} \mathrm{H}\right]$ palmitate, $\left[\mathrm{U}_{-}{ }^{14} \mathrm{C}\right]$ glucose, $\left[5^{-3} \mathrm{H}\right]$ glucose, or $\left[\mathrm{U}_{-}{ }^{14} \mathrm{C}\right]$ lactate, respectively, were added to the Krebs-Henseleit solution. At $30 \mathrm{~min}$ of the $60 \mathrm{~min}$ perfusion, $100 \mu \mathrm{U} / \mathrm{mL}$ insulin was added to the KrebsHenseleit solution. Glucose and lactate oxidation rates were assessed by measuring ${ }^{14} \mathrm{CO}_{2}$ production. Palmitate oxidation and glycolysis rates were assessed by measuring ${ }^{3} \mathrm{H}_{2} \mathrm{O}$ production. Proton production was determined by subtracting the glucose oxidation rate from the glycolysis rate and multiplying the result by 2 (Liu et al. 2002). Mechanical function was measured using a Powerlab acquisition system and a Transonic flow meter and probes were placed in the preload and afterload lines to measure cardiac output and aortic flow. Cardiac work (joules $/ \mathrm{min} / \mathrm{g}$ dry weight) was calculated by subtracting preload pressure from peak systolic pressure which was then multiplied by cardiac output and normalized against the heart dry weight. At the end of the aerobic perfusion protocol, hearts were immediately frozen in liquid $\mathrm{N}_{2}$ and stored at $-80{ }^{\circ} \mathrm{C}$ (Barr and Lopaschuk 1997; Ussher et al. 2009).

\section{Western blot analysis}

Standard western blot procedures were followed. Briefly, frozen ventricular tissue was homogenized for $30 \mathrm{~s}$ in buffer containing $50 \mathrm{mM}$ Tris $\mathrm{HCl}, 1 \mathrm{mM}$ EDTA, 10\% glycerol, $0.02 \%$ Brij-35, $1 \mathrm{mM}$ dithiothreitol (DTT), and protease and phosphatase inhibitors (Sigma). The homogenate was left on ice for $10 \mathrm{~min}$ and then centrifuged at $10,000 \mathrm{x} \mathrm{g}$ for $20 \mathrm{~min}$. Protein concentration of supernatant was determined using a Bradford protein assay. SDS-polyacrylamide gel electrophoresis was used and protein was transferred onto a $0.45 \mu \mathrm{m}$ nitrocellulose membrane. Membranes were blocked with $5 \%$ fat free milk for $1 \mathrm{~h}$ and probed with primary antibodies in 5\% BSA overnight. Primary antibodies included pyruvate dehydrogenase (PDH; Cell Signaling 2784), phosphoSer293 PDH (Calbiochem AP1062), phosphoThr389 p70S6K (Cell Signaling 9206), p70S6K (Cell Signaling 9202), phosphoglycerate mutase 1 (PGAM1) (Cell Signaling 7534), hydroxyacyl coenzyme A dehydrogenase (HADH) (Abcam ab93172), GLUT1 (Santa Cruz 1605), GLUT4 (Santa Cruz 1606), lactate dehydrogenase A (LDHA) (Santa Cruz 27,230), mitochondrial pyruvate carrier 1 (MPC1) (Cell Signaling 14,462), MPC2 (Cell Signaling 46,141), cytochrome c (Santa Cruz 8385) and hypoxia inducible factor (HIF) $1 \alpha$ (Novus Biologicals 100-105)). Membranes were then washed $4 \times 5 \mathrm{~min}$ in Tris-buffered saline tween, probed with secondary antibody, goat antirabbit (Santa Cruz 2054), goat antimouse (Santa Cruz 2055), or donkey antigoat (Jackson Immunoresearch 705,035,003), and again washed $4 \times 5 \mathrm{~min}$ in Tris-buffered saline tween. Protein bands were then visualized with enhanced chemiluminescence (Perkin Elmer). Quantification was performed using Image J.

\section{Statistical analysis}

Values are presented as mean \pm SEM. One Way Analysis of Variance (ANOVA) with Bonferroni posthoc test was performed or Kruskal-Wallis test with Dunn's Multiple Comparison test was performed as appropriate using Prism software to determine statistical significance. Differences are considered significant if $\mathrm{p}<0.05 . \mathrm{n}$ size is indicated in the figure legends.

\section{Results}

Feeding a high salt diet (HSD) to Dahl salt-sensitive rats results in the progressive development of hypertrophy and diastolic dysfunction

When Dahl salt-sensitive rats were fed a HSD, a progressive decrease in diastolic function was observed over a 9 week period. In vivo echocardiography of the hearts showed a significant decrease in E'/A' (a measure of diastolic dysfunction) by 6 weeks following initiation of the HSD (Fig. 1a). In contrast, systolic function was largely preserved, with \%EF decreasing slightly by 9 weeks (Fig. 1b), and \%FS remaining unchanged (Table 1). This suggests that the rats developed HFpEF prior to developing heart failure with reduced ejection fraction (HFrEF). In isolated working hearts obtained from rats at each time period studied a progressive decrease in cardiac work was observed over the 9 week period (Fig. 1c), primarily due to a decrease in cardiac output (Table 2). This time frame for heart failure development agrees with other studies examining the development of heart failure in DSS rats fed a HSD (Rapp and Dene 1985; Klotz et al. 2006). The progressive development of diastolic dysfunction in the Dahl salt-sensitive rats following administration of the HSD was accompanied by an increase in LV mass (Fig. 1d), as well as an increase in IVSd and LVPWd (Fig. 1e, Table 1). At the same time the phosphorylation of p70S6k increased suggesting a stimulation of the mTOR pathway (which promotes cardiac hypertrophy) (Fig. 1f). 

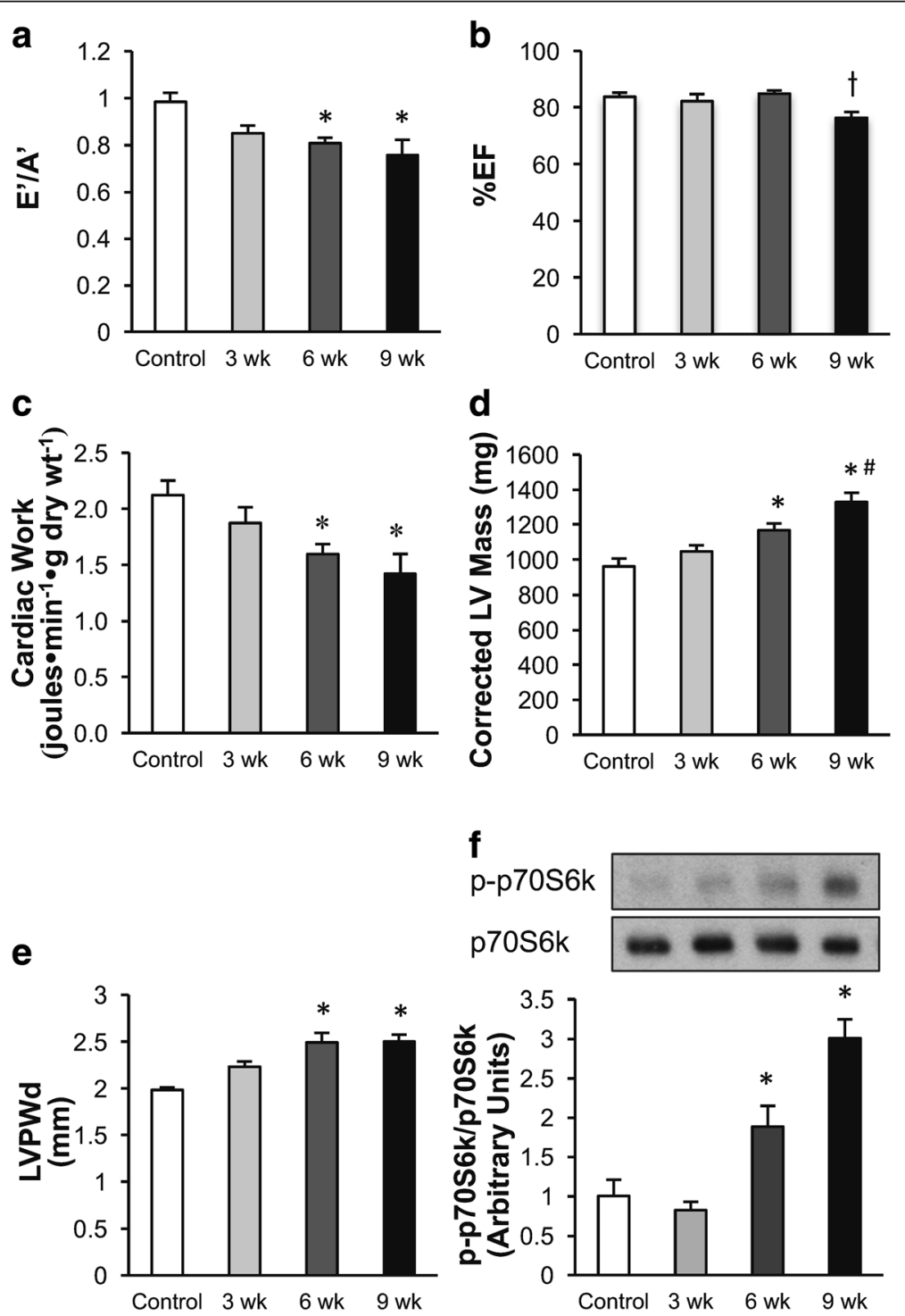

Fig. 1 Time dependent effects of a high salt diet (HSD) on Dahl salt-sensitive rat cardiac function and hypertrophy. a Diastolic dysfunction was measured by E'/A'. b \%EF was measured by echocardiography. c Cardiac work was measured during the isolated working heart perfusions. d-e Cardiac hypertrophy was measured by corrected LV mass and LVPWd using echocardiography. f Cardiac p-p70S6K/p70S6K protein expression was assessed in hearts obtained at each of the time periods studied. Measurements were made in Dahl salt-sensitive rats fed a low salt diet, $0.3 \% \mathrm{NaCl}$ (Control) or a HSD, 8\% NaCl, for 3, 6, or 9 weeks. $\mathrm{n}=5-8 * p<0.05$ compared to Control. \# $p<0.05$ compared to 3 weeks. t $p<0.05$ compared to 6 weeks. Values shown as mean \pm SEM

The development of diastolic dysfunction is accompanied by a decrease in cardiac mitochondrial oxidative metabolism in dahl salt-sensitive rats

Rates of overall energy metabolism were measured in isolated working hearts obtained at 3 weeks, 6 weeks, and 9 weeks of the HSD. A progressive decrease in fatty acid oxidation rates was observed following the HSD (Fig. 2a), although a significant decrease in fatty acid oxidation rates did not occur until 9 weeks of the HSD. In the presence of insulin, a similar time-dependent decrease in fatty acid oxidation was also observed
(Table 3). In contrast, there was no change in glucose oxidation rates (Fig. 2b) or lactate oxidation rates (Fig. 2c) during the development of diastolic dysfunction, regardless of whether insulin was present (Table 3) or absent. The primary source of overall cardiac ATP production in all hearts originated from fatty acid oxidation (Fig. 2d). As a result, a decrease in overall cardiac ATP production rates were observed by 9 weeks of the HSD, which was primarily due to the observed decrease in fatty acid oxidation rates (Fig. 2d). 
Table 1 Effect of a high salt diet (HSD) on in vivo cardiac function in Dahl salt-sensitive rats

\begin{tabular}{|c|c|c|c|c|}
\hline & Control & 3 weeks & 6 weeks & 9 weeks \\
\hline EF (\%) & $83.79 \pm 1.44$ & $82.21 \pm 2.50$ & $84.91 \pm 1.14$ & $76.21 \pm 2.19^{\top}$ \\
\hline FS (\%) & $54.60 \pm 1.69$ & $53.12 \pm 2.57$ & $54.45 \pm 2.39$ & $47.64 \pm 2.34$ \\
\hline LV Mass Corrected & $962.88 \pm 43.45$ & $1045.85 \pm 36.11$ & $1167.85 \pm 38.96^{*}$ & $1328.94 \pm 52.37^{* \#}$ \\
\hline IVSd (mm) & $1.97 \pm 0.05$ & $2.23 \pm 0.06^{*}$ & $2.40 \pm 0.07^{*}$ & $2.50 \pm 0.07^{* \#}$ \\
\hline LVIDd (mm) & $7.91 \pm 0.12$ & $7.32 \pm 0.14^{*}$ & $7.45 \pm 0.09$ & $7.52 \pm 0.20$ \\
\hline LVPWd (mm) & $1.98 \pm 0.03$ & $2.23 \pm 0.06$ & $2.49 \pm 0.10^{*}$ & $2.50 \pm 0.08^{*}$ \\
\hline IVSs (mm) & $3.40 \pm 0.05$ & $3.67 \pm 0.10$ & $3.68 \pm 0.09$ & $3.68 \pm 0.15$ \\
\hline LVIDs (mm) & $3.87 \pm 0.18$ & $3.46 \pm 0.23$ & $3.40 \pm 0.09$ & $4.25 \pm 0.08$ \\
\hline LVPWs (mm) & $3.45 \pm 0.12$ & $3.68 \pm 0.11$ & $3.77 \pm 0.08$ & $3.65 \pm 0.13$ \\
\hline LV Vol;d & $335.75 \pm 10.95$ & $283.11 \pm 12.35^{*}$ & $294.62 \pm 7.78$ & $301.74 \pm 17.16$ \\
\hline LV Vol;s & $51.99 \pm 6.28$ & $45.76 \pm 6.96$ & $47.51 \pm 3.37$ & $81.30 \pm 3.62^{* \# T}$ \\
\hline$E / E^{\prime}$ & $19.04 \pm 2.18$ & $20.49 \pm 1.95$ & $18.82 \pm 1.22$ & $18.29 \pm 1.51$ \\
\hline$E / A$ & $1.60 \pm 0.11$ & $1.64 \pm 0.07$ & $1.33 \pm 0.15$ & $1.40 \pm 0.20$ \\
\hline$E^{\prime} / A^{\prime}$ & $0.98 \pm 0.04$ & $0.85 \pm 0.03$ & $0.81 \pm 0.02^{*}$ & $0.76 \pm 0.07^{*}$ \\
\hline Tei Index & $0.65 \pm 0.03$ & $0.72 \pm 0.04$ & $0.78 \pm 0.05$ & $0.76 \pm 0.05$ \\
\hline$E^{\prime}$ & $54.23 \pm 6.85$ & $54.68 \pm 4.61$ & $46.79 \pm 2.14$ & $38.26 \pm 3.49$ \\
\hline IVRT (ms) & $24.32 \pm 0.60$ & $22.68 \pm 0.68$ & $27.97 \pm 1.76$ & $28.13 \pm 1.63$ \\
\hline IVCT (ms) & $15.69 \pm 0.90$ & $16.88 \pm 1.29$ & $15.81 \pm 1.48$ & $17.47 \pm 1.43$ \\
\hline HR (bpm) & $365.25 \pm 6.61$ & $375.88 \pm 7.71$ & $367.63 \pm 10.98$ & $349.75 \pm 16.68$ \\
\hline L Kidney/TL (g/cm) & $0.31 \pm 0.01$ & $0.34 \pm 0.01$ & $0.34 \pm 0.01$ & $0.38 \pm 0.03^{*}$ \\
\hline
\end{tabular}

In vivo cardiac function was measured via echocardiography in Dahl salt-sensitive rats fed a low salt diet, $0.3 \% \mathrm{NaCl}(\mathrm{Control})$, or a high salt diet, $8 \% \mathrm{NaCl}$, for 3,6 , or 9 weeks. $n=5-8{ }^{*} p<0.05$ compared to Control. ${ }^{*} p<0.05$ compared to Control. ${ }^{*} p<0.05$ compared to 3 weeks. ${ }^{\top} p<0.05$ compared to 6 weeks. $n=6-9$ Values shown as mean \pm SEM \% Ejection fraction (\%EF); \% Fractional shortening (\%FS); left ventricle (LV); Interventricular septum end diastole (IVSd); LV internal diameter end diastole (LVIDd); LV posterior wall thickness end diastole (LVPWd); Interventricular septum end systole (IVSs); LV internal diameter end systole (LVIDs); LV posterior wall thickness end systole (LVPWs); LV volume end diastole (LV Vol;d); LV volume end systole (LV Vol;s); Isovolumetic relaxation time (IVRT); Isovolumetic contraction time (IVCT); Tibia length (TL); Heart rate (HR)

Decreased glycolysis is an early energy metabolic change in hearts from Dahl salt-sensitive rats fed a HSD

The earliest energy metabolic change that occurred in Dahl-sensitive rats fed a HSD was an increase in glycolysis, which had already increased over $300 \%$ by 3 weeks of the HSD (Fig. 2e). This increase was also observed when insulin was present in the perfusate (Table 3), and prior to the onset of either HFpEF or HFrEF (Fig. 1). Since the increase in glycolysis during the development of diastolic dysfunction was not accompanied by an increase in glucose oxidation (Fig. 2b), an uncoupling of glycolysis from glucose oxidation occurred, resulting in a significant increase in proton production, even by 3 weeks of the HSD (Fig. 2f). This increased uncoupling of glycolysis and glucose oxidation and rise in proton production persisted in hearts perfused in the presence of insulin (Table 3). Since the uncoupling of glycolysis and glucose oxidation and elevation in proton

Table 2 Effect of a high salt diet (HSD) on cardiac function ex vivo in Dahl salt-sensitive rats

\begin{tabular}{|c|c|c|c|c|}
\hline & Control & 3 weeks & 6 weeks & 9 weeks \\
\hline Heart rate (beats $\bullet \mathrm{min}^{-1}$ ) & $277.9 \pm 7.3$ & $290.8 \pm 8.6$ & $278.7 \pm 6.1$ & $266.9 \pm 22.9$ \\
\hline Peak Systolic Pressure (mmHg) & $109.9 \pm 1.0$ & $107.8 \pm 2.6$ & $110.8 \pm 1.7$ & $107.5 \pm 6.7$ \\
\hline Developed Pressure (mmHg) & $41.9 \pm 2.8$ & $38.5 \pm 3.8$ & $42.6 \pm 2.2$ & $43.1 \pm 7.0$ \\
\hline Cardiac Output $\left(\mathrm{m} / \bullet \mathrm{min}^{-1}\right)$ & $50.4 \pm 2.2$ & $45.7 \pm 1.9$ & $41.6 \pm 2.2$ & $38.5 \pm 3.1^{*}$ \\
\hline Coronary Flow $\left(\mathrm{ml} \bullet \mathrm{min}^{-1}\right)$ & $26.5 \pm 2.8$ & $23.9 \pm 0.5$ & $22.3 \pm 0.7$ & $20.8 \pm 1.5$ \\
\hline Cardiac Work (joules $\min ^{-1} \bullet \mathrm{g}$ dry weight ${ }^{-1}$ ) & $2.1 \pm 0.1$ & $1.9 \pm 0.1$ & $1.6 \pm 0.1^{*}$ & $1.4 \pm 0.2^{*}$ \\
\hline
\end{tabular}

Cardiac function was measured in isolated working hearts from Dahl salt-sensitive rats fed a low salt diet, $0.3 \% \mathrm{NaCl}$ (Control), or a high salt diet, $8 \% \mathrm{NaCl}$, for 3,6 , or 9 weeks. $n=3-5 * p<0.05$ compared to Control. Values shown as mean \pm SEM 


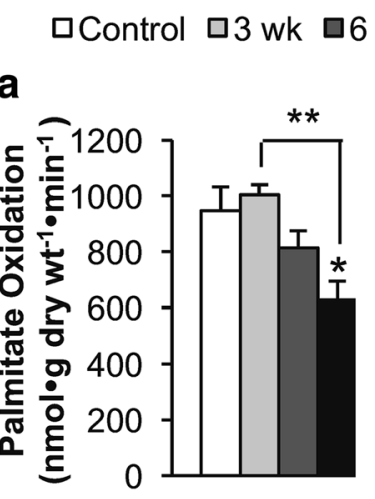

b

C

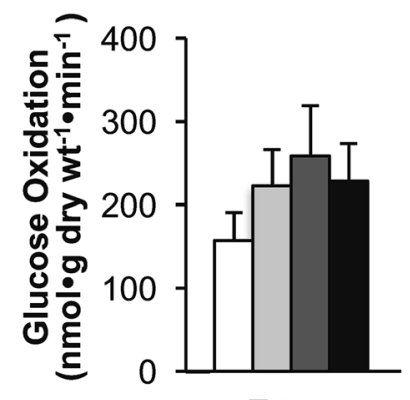

d

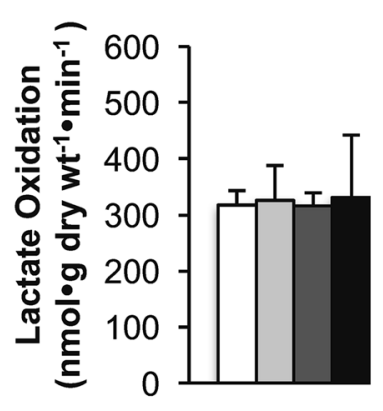

T140 14 Glycolysis

L Lactate Oxidation

- Glucose Oxidation

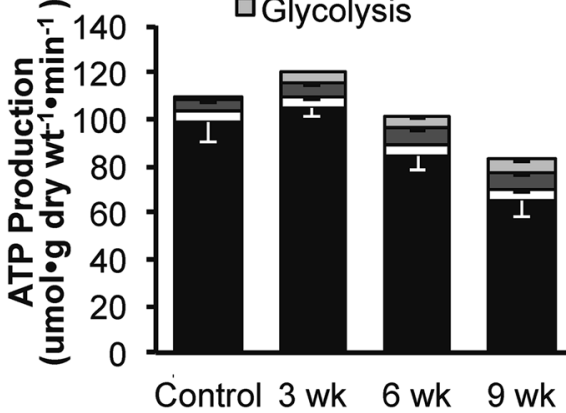

e

f
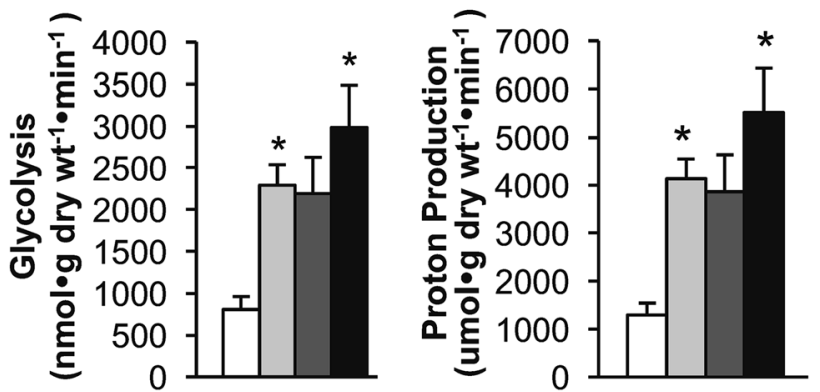

Fig. 2 Time dependent effects of a high salt diet (HSD) on palmitate oxidation, glucose oxidation, glycolysis, and lactate oxidation in Dahl salt-sensitive rat hearts. a Palmitate oxidation, (b) Glucose oxidation, (c) Lactate oxidation, (d) ATP production, (e) Glycolysis, and (f) Proton production were measured in hearts from Dahl salt-sensitive rats fed a low salt diet, $0.3 \% \mathrm{NaCl}$ (Control) or a HSD, $8 \% \mathrm{NaCl}$, for 3, 6, or 9 weeks. Energy metabolic rates were assessed in isolated working hearts. Proton production was calculated based on glycolysis and glucose oxidation rates. Contribution to ATP production was calculated from the metabolic rates assessed using the isolated working heart perfusion in Dahl salt-sensitive rats. $n=3-5 * p<0.05$ compared to Control. Values shown as mean \pm SEM

production occurs early during the development of diastolic dysfunction, a possible causal link between the uncoupling of glycolysis and glucose oxidation and the development of HFpEF may exist.

Even though glycolysis rates in the hearts remained elevated as HFrEF developed (i.e. by 9 weeks of the HSD), the increase in ATP production originating from glycolysis did not compensate for the decrease in ATP production that occurred as a result of the decrease in fatty acid oxidation (Fig. 2d, Table 3).
Increased GLUT1 expression may contribute to the increased uncoupling of glycolysis from glucose oxidation observed during the development of diastolic dysfunction

Examination of the expression of various proteins involved in glucose metabolism indicated that a change in glucose transport may contribute to the rise in glycolysis seen during the development of diastolic dysfunction. A progressive increase in GLUT1 expression was seen in Dahl salt-sensitive rat hearts during the HSD (Fig. 3a 
Table 3 Effect of insulin $(100 \mu \mathrm{U} / \mathrm{ml})$ on cardiac metabolism in Dahl salt-sensitive rats fed a high salt diet (HSD)

\begin{tabular}{lllll}
\hline & Control & 3 weeks & 6 weeks & 9 weeks \\
\hline Glycolysis & $1231.3 \pm 234.8$ & $2390.9 \pm 390.4$ & $2090.6 \pm 647.2$ & $2982.2 \pm 734.1$ \\
Glucose Oxidation & $312.2 \pm 20.6$ & $519.0 \pm 119.7$ & $464.8 \pm 113.6$ & $493.0 \pm 166.7$ \\
Palmitate Oxidation & $983.5 \pm 47.5$ & $931.0 \pm 38.2$ & $796.9 \pm 43.8$ & $616.3 \pm 56.9^{*}$ \\
Lactate Oxidation & $377.0 \pm 46.2$ & $426.5 \pm 95.0$ & $339.6 \pm 62.4$ & $275.0 \pm 113.7$ \\
Proton Production & $1838.3 \pm 454.1$ & $3743.8 \pm 558.6$ & $3251.6 \pm 1073.8$ & $4978.3 \pm 1197.6$ \\
ATP Production & & & & $6.0 \pm 1.5$ \\
Glycolysis & $2.5 \pm 0.5$ & $4.8 \pm 0.8$ & $4.2 \pm 1.3$ & $13.3 \pm 4.8$ \\
Glucose Oxidation & $9.1 \pm 0.6$ & $15.1 \pm 3.5$ & $82.9 \pm 4.6$ & $64.1 \pm 5.9^{*}$ \\
Palmitate Oxidation & $102.3 \pm 4.9$ & $96.8 \pm 4.0$ & $4.9 \pm 0.9$ & $106.4 \pm 10.2$ \\
Lactate Oxidation & $5.5 \pm 0.7$ & $6.2 \pm 1.4$ & $4.0 \pm 1.6$ \\
Total & $115.6 \pm 3.3$ & $122.8 \pm 6.2$ & $88.3 \pm 11.0$ \\
\hline
\end{tabular}

Energy metabolic rates (nmoløg dry $\mathrm{wt}^{-1} \bullet \mathrm{min}^{-1}$ ) were measured during the working heart perfusion. Contribution to ATP production $\left(\mu \mathrm{mol} \bullet \mathrm{g}\right.$ dry wt ${ }^{-1} \bullet \mathrm{min}^{-1}$ ) was calculated from the metabolic rates assessed via the isolated working heart perfusion in Dahl salt-sensitive rats. These results are from Dahl salt-sensitive rats fed a low salt diet, $0.3 \% \mathrm{NaCl}$ (Control), or a high salt diet, $8 \% \mathrm{NaCl}$, for 3,6 , or 9 weeks. $\mathrm{n}=3-5 * p<0.05$ compared to Control. \# $p<0.05$ compared to 3 weeks. Values shown as mean \pm SEM

and b). Since GLUT1 mediates glucose uptake independent of insulin, it suggests that increased GLUT1 expression may be involved in the elevated glycolysis rates observed even when hearts were perfused without insulin (Fig. 2e). While PGAM1 and GLUT4 (the insulindependent glucose transporter) expression were not significantly altered during the development of diastolic dysfunction, LDHA was significantly increased after 3 weeks on the HSD (Fig. 3d). This isoform of LDH favors the conversion of pyruvate to lactate. This suggests that LDHA may contribute to the initial increase in uncoupling of glycolysis and glucose oxidation observed in response to the HSD. HIF1 $\alpha$, a transcription factor regulating glycolysis, was not altered by the HSD (Fig. 3e).

We also examined the expression of mitochondrial enzymes that might contribute to the changes in cardiac energy metabolism observed in response to the HSD. No significant changes were observed in PDH expression, the rate-limiting enzyme for glucose oxidation (Fig. 4a). While phosphorylation of PDH by PDH kinase decreases PDH activity, we observed no change in pPDH during the development of diastolic dysfunction (Fig. 4a and b). This lack of change in pPDH correlates with the lack of change in glucose oxidation rates during the development of diastolic dysfunction (Fig. 2b). We also looked at the expression of the mitochondrial pyruvate carrier. Interestingly, MPC1 expression was increased after 6 weeks on the HSD, but MPC2 expression was not significantly altered (Fig. 4c and d). In addition, cytochrome c protein expression was not significantly altered (Fig. 4f). Since acetylation has been shown to regulate mitochondrial oxidative metabolism we also assessed the effect of the HSD on overall acetylation. However, overall lysine acetylation was not significantly altered in hearts of Dahl salt-sensitive rats fed a HSD (Additional file 1).

\section{Discussion}

Alterations in cardiac energy metabolism are thought to be an important contributor to the severity of heart failure. However, there is confusion as to what changes in cardiac energy metabolism occur in heart failure, although it is generally believed that in HFrEF fatty acid metabolism decreases while overall glucose metabolism increases (Kato et al. 2010; Ingwall 2007; Davila-Roman et al. 2002). In this study we directly determined for the first time that the earliest cardiac energy metabolic changes that occurs in HFpEF is a dramatic increase in glycolysis. This metabolic change occurs prior to the development of HFpEF. Of interest, is that this increase in glycolysis occurs without a parallel change in glucose oxidation, which results in increased uncoupling of glycolysis and glucose oxidation. This is notable as previously published research has shown that increasing this uncoupling of glycolysis and glucose oxidation can result in intracellular acidosis, which can impair cardiac function (Liu et al. 1996; Liu et al. 2002; Steenbergen et al. 1977). An increased uncoupling of glycolysis and glucose oxidation has also been reported in other more severe models of heart failure such as in rodent hearts subjected to coronary artery ligation (CAL) (Masoud et al. 2014). Further, in one study abdominal aortic banding induced changes in cardiac metabolite levels indicative of increased uncoupling of glycolysis and glucose oxidation due to elevated glycolysis (Seymour et al. 2015). Our data also confirms that fatty acid oxidation decreases in HFrEF. There is evidence indicating that a reduction in fatty acid oxidation may contribute to diastolic dysfunction. For example, it was recently reported that overexpressing Acetyl Coenzyme A Carboxylase both prevented diastolic dysfunction and reduced cardiac fatty acid oxidation in mice treated with Angiotensin II (Choi et al. 2016; 


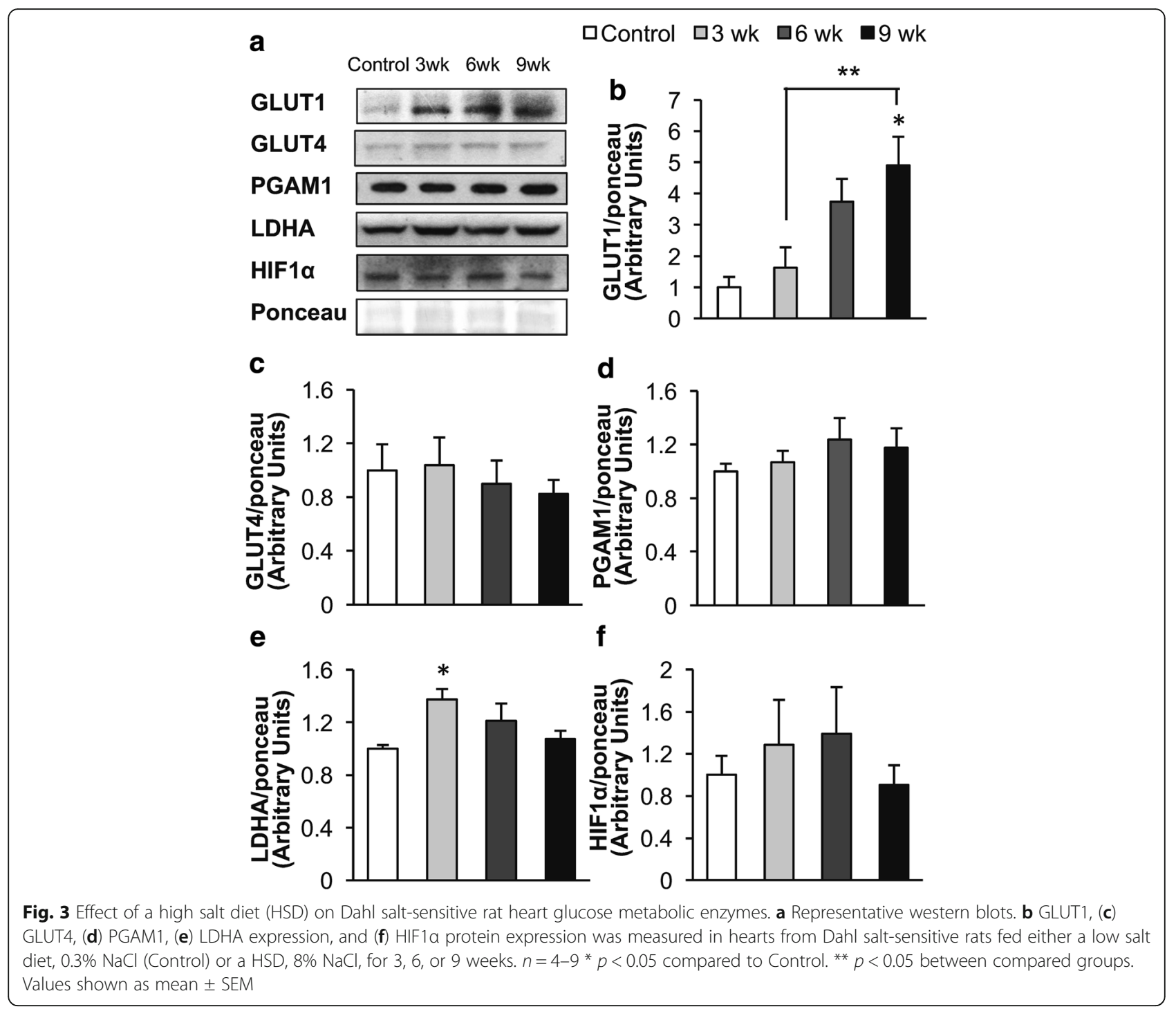

Helge et al. 1996). However, of importance is that the decrease in fatty acid oxidation seen in the failing heart in our study did not precede the onset of diastolic dysfunction. As a result, it is unlikely that a decrease in fatty acid oxidation is contributing to the early development of diastolic dysfunction in heart failure.

While it is often cited that in heart failure the heart switches from fatty acid to glucose metabolism, our data suggests that it is more accurate to suggest that a decrease in overall cardiac mitochondrial oxidative metabolism occurs in HFrEF, accompanied by a relative increase in glycolysis. Despite glucose oxidation being the major source of glucose derived ATP production, glucose oxidation rates are not increased during the development of systolic dysfunction (Kato et al. 2010; Lopaschuk et al. 2010; Zhabyeyev et al. 2013; Zhang et al. 2013; Liu et al. 1996; Liu et al. 2002). In fact, in mouse models of HFrEF, we actually observed a decrease in glucose oxidation rates (Zhabyeyev et al. 2013; Zhang et al. 2013).

There are several potential explanations for this rise in cardiac glycolysis in diastolic dysfunction. One possibility may be that the overall decrease in mitochondrial oxidative metabolism (which we have previously reported in hearts from HFpEF mice), results in a compensatory rise in glycolysis (Kato et al. 2010; Beer et al. 2002; Masoud et al. 2014; Zhang et al. 2013; Neubauer 2007). The decrease in cardiac ATP production after 9 weeks on a HSD appears to be solely due to a drop in fatty acid oxidation, as glucose and lactate oxidation remained unchanged. In an effort to determine what was responsible for this decrease in fatty acid oxidation we examined the expression of enzymes involved in fatty acid oxidation and mitochondrial oxidative metabolism. It has been previously reported that the cardiac expression of proteins involved in fatty acid oxidation and overall mitochondrial oxidative 


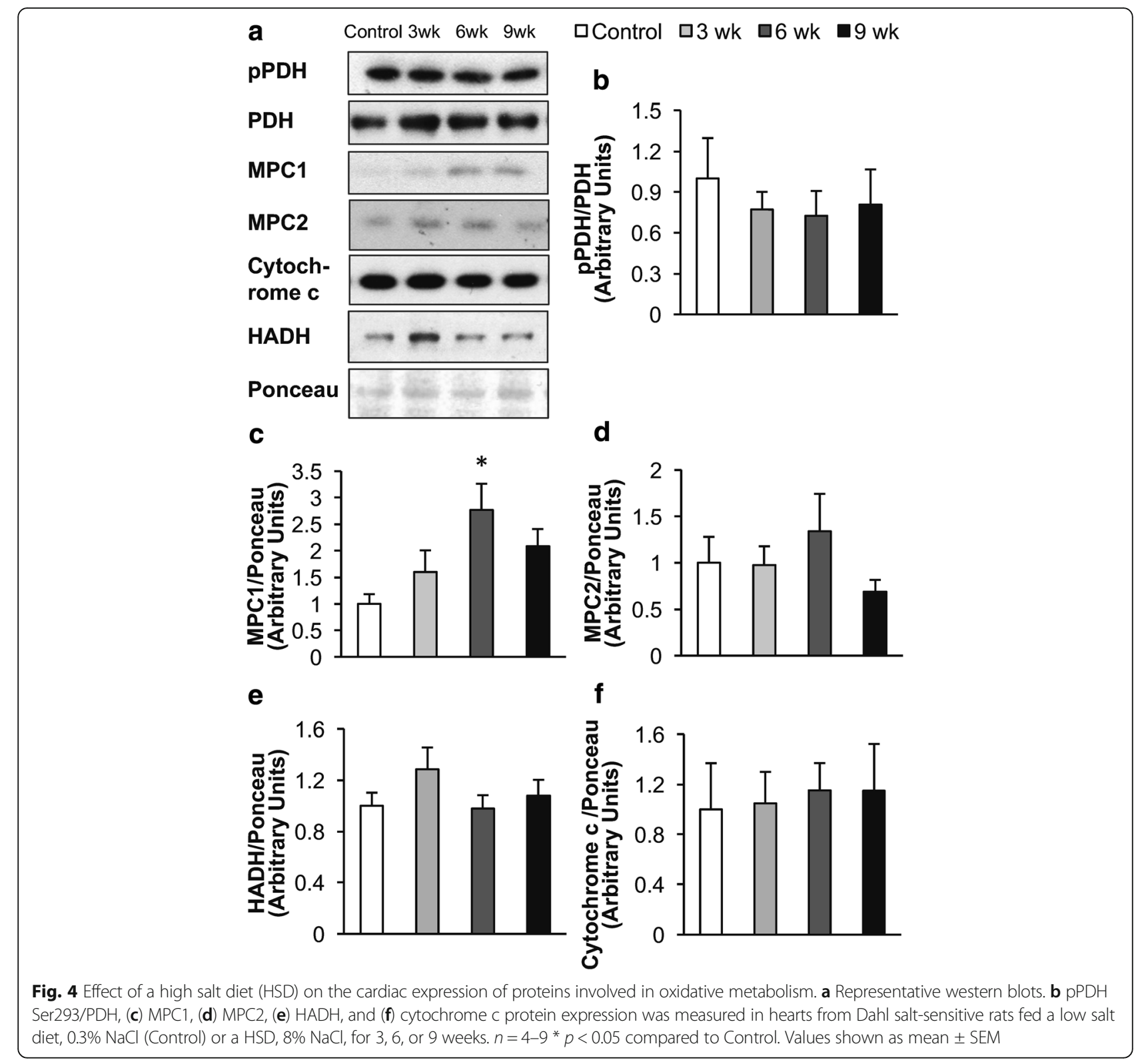

metabolism are decreased in severe heart failure (Zhang et al. 2013; Lai et al. 2014). However, we did not observe a change in the expression of $ß$-hydroxyacyl CoA dehydrogenase or cytochrome $\mathrm{c}$ in the heart (Fig. 4), enzymes involved in fatty acid oxidation and mitochondrial oxidative metabolism, respectively. Alternatively, the decrease in cardiac work may be lowering cardiac fatty acid oxidation rates. Cardiac fatty acid oxidation rates remained unchanged during the development of diastolic dysfunction when normalized against cardiac work (Control, 410.4 \pm 41.8 ; 3 weeks, $498.5 \pm 38$; 6 weeks, $502.2 \pm 31.4$; 9 weeks, $388.0 \pm 16.1 \mathrm{nmol} \bullet \mathrm{g}$ dry $\mathrm{wt}^{-1} \bullet \mathrm{min}^{-1}$ ) (Online Resource $1)$. While it is possible that a reduction in fatty acid oxidation is causing the decrease in cardiac function, we speculate that the reduction in cardiac fatty acid oxidation is a consequence of reduced cardiac work.

Increased capacity for glycolysis or glucose transport may also be responsible for the rise in glycolysis during development of diastolic dysfunction and heart failure. Although we did not observe a change in the expression of the glycolytic enzyme PGAM1, we did find an increase in LDHA protein expression after 3 weeks on the HSD and a later rise in GLUT1 protein expression (Fig. 4). LDH isoforms that contain LDHA are more likely to convert pyruvate to lactate, as opposed to catalyzing the opposite reaction. Therefore, increased LDHA 
protein expression could be contributing to the early rise in glycolysis and increased uncoupling of glycolysis and glucose oxidation in response to the HSD.

Increased cardiac GLUT1 expression may also contribute to the rise in glycolysis. In support of this, glycolysis is elevated in hearts overexpressing GLUT1 and is decreased in hearts lacking GLUT1 (Liao et al. 2002; Pereira et al. 2014). However, studies that regulate GLUT1 expression report mixed results on the role of GLUT1 in the development of heart failure. While overexpression of GLUT1 has been reported to prevent pressure overload induced heart failure in mouse hearts, deletion of GLUT1 does not affect the rate of development of pressure overload induced heart failure. The GLUT1 knockout mouse has elevated fatty acid oxidation and reduced glucose oxidation, which would be expected to decrease cardiac efficiency and may explain why these hearts are not resistant pressure overload induced heart failure (Pereira et al. 2014). However, the results from these two studies do not preclude the possibility that a more acute up-regulation of GLUT1 expression could increase glycolysis and impair cardiac function.

Based on these results we hypothesize that stimulating glucose oxidation may be a promising strategy for treating and potentially preventing the development of HFpEF. As mentioned earlier, stimulating cardiac glucose oxidation is associated with an increase in cardiac efficiency and an improvement in cardiac function (Kato et al. 2010; Masoud et al. 2014; Yamashita et al. 2009; Ussher et al. 2012; Stanley et al. 2005; Lopaschuk et al. 2003; Dyck et al. 2006; Dyck et al. 2004). Stimulating cardiac glucose oxidation can also be beneficial in the context of obesity and diabetes, which can lead to heart failure (Ussher et al. 2009; Lewis et al. 2016; Nicholl et al. 1991). Furthermore, treatment of Dahl salt sensitive rats with dichloroacetate (DCA) decreases plasma lactate levels (an indirect indication of elevated glycolysis), and improves cardiac function (Kato et al. 2010). However, in this study intervention with DCA was at a later stage of heart failure development, which was also associated with changes in systolic function. In the future it will be important to determine if stimulating glucose oxidation with more potent PDK inhibitors can lessen or even prevent the development of HFpEF.

\section{Conclusions}

This study directly characterized the changes in cardiac energy metabolism that occur in diastolic dysfunction. We demonstrate that the earliest cardiac metabolic change that occurs during the development of diastolic dysfunction is an increase in glycolysis, with no change in carbohydrate or fatty acid oxidation. The rise in glycolysis resulted in increased uncoupling of glycolysis and glucose oxidation and an increased proton production, which occurs early during the development of diastolic dysfunction. Our findings combined with previous work suggest that the coupling of glycolysis and glucose oxidation is important in maintaining normal cardiac function and may contribute to the development of HFpEF. While these results suggest that decreasing the uncoupling of glycolysis and glucose oxidation may be a promising strategy for the treatment of heart failure, more work is needed to determine if therapeutically improving the coupling of glycolysis and glucose oxidation can treat HFpEF.

\section{Additional file}

Additional file 1: Figure S1. Effect of a high salt diet (HSD) on overall protein acetylation. Total protein acetylation levels were measured in hearts from Dahl salt-sensitive rats fed a low salt diet, $0.3 \% \mathrm{NaCl}$ (Control) or a HSD, $8 \% \mathrm{NaCl}$, for 3,6 , or 9 wk. $n=6-9$ Values shown as mean \pm SEM. (TIFF $3225 \mathrm{~kb}$ )

\section{Abbreviations}

\%EF: \% Ejection fraction; \%FS: \% Fractional shortening; ANOVA: One Way Analysis of Variance; ATP: Adenosine triphosphate; Bpm: beats per minute; BSA: Bovine serum albumin; CAL: Coronary artery ligation; DCA: Dichloroacetate; DTT: Dithiothreitol; GLUT1: Glucose transporter 1; GLUT4: Glucose transporter 4; HADH: Hydroxyacyl coenzyme A dehydrogenase; HF: Heart failure; HFpEF: Heart failure with preserved ejection fraction; HFrEF: Heart failure with reduced ejection fraction; HIF1a: Hypoxia inducible factor 1a; HR: Heart rate; HSD: High salt diet; IVRT: Isovolumetric relaxation time; IVSd: Interventricular septum end diastole; IVSs: Interventricular septum end systole; LDHA: Lactate dehydrogenase A; LV Vol,d: Left ventricle volume end diastole; LV Vol;s: Left ventricle volume end systole; LV: Left ventricle; LVIDd: Left ventricle internal diameter end diastole; LVIDs: Left ventricle internal diameter end systole; LVPWd: Left ventricle posterior wall thickness end diastole; LVPWs: Left ventricle posterior wall thickness end systole; MPC1: Mitochondrial pyruvate carrier 1; MPC2: Mitochondrial pyruvate carrier 2; PDH: Pyruvate dehydrogenase; PGAM1: Phosphoglycerate mutase 1

\section{Acknowledgements}

We thank the Cardiovascular Research Centre Core for assessment of in vivo cardiac function.

\section{Funding}

This work was supported by an Alberta Innovates - Health Solutions Interdisciplinary Team Grant via Alberta Heart; and the Canadian Institutes of Health Research [to G.D.L, and to J.R.B.D]. G.D.L is an Alberta Innovates - Health Solutions Scientist and N.F. holds an Alberta Innovates - Health Solutions studentship.

Availability of data and materials

Data in this study are included in this published article.

\section{Authors' contributions}

NF designed the study, collected, analyzed and interpreted the data, and wrote the manuscript. JLL, AF, CSW, and WW collected, analyzed, and interpreted data for the study. JRBD revised the manuscript and contributed to the discussion. GDL designed the study, contributed to the discussion, and wrote the manuscript. The final manuscript was approved by all authors.

\section{Ethics approval}

All procedures on animals were approved by the University of Alberta Health Sciences Animal Welfare Committee and conformed to the Canadian

Council on Animal Care guidelines (Canadian Council on Animal Care 2017).

Consent for publication

Not applicable 


\section{Competing interests}

The authors declare that they have no competing interests.

\section{Publisher's Note}

Springer Nature remains neutral with regard to jurisdictional claims in published maps and institutional affiliations.

\section{Received: 13 January 2018 Accepted: 13 February 2018}

\section{Published online: 15 March 2018}

\section{References}

Allard MF, Schonekess BO, Henning SL, English DR, Lopaschuk GD. Contribution of oxidative metabolism and glycolysis to ATP production in hypertrophied hearts. Am J Physiol Heart Circ Physiol. 1994;267:H742-H50.

Barr RL, Lopaschuk GD. Direct measurement of energy metabolism in the isolated working rat heart. J Pharmacol Toxicol Methods. 1997;38:11-7.

Beer M, Seyfarth T, Sandstede J, Landschutz W, Lipke C, Kostler H, et al. Absolute concentrations of high-energy phosphate metabolites in normal, hypertrophied, and failing human myocardium measured noninvasively with (31)P-SLOOP magnetic resonance spectroscopy. J Am Coll Cardiol. 2002;40:1267-74.

Bonnet S, Archer SL, Allalunis-Turner J, Haromy A, Beaulieu C, Thompson R, et al. A mitochondria-K+ channel axis is suppressed in cancer and its normalization promotes apoptosis and inhibits cancer growth. Cancer Cell. 2007:11:37-51.

Canadian Council on Animal Care. (2017) Guide to the care and use of experimental animals. 2nd. Ottawa, Ontario. [cited 2017 Sept 16]. Available from: http://www.ccac.ca/.

Chesnais JM, Coraboeuf E, Sauviat MP, Vassas JM. Sensitivity to H, li and mg ions of the slow inward sodium current in frog atrial fibres. J Mol Cell Cardiol. 1975; $7: 627-42$

Choi YS, de Mattos AB, Shao D, Li T, Nabben M, Kim M, et al. Preservation of myocardial fatty acid oxidation prevents diastolic dysfunction in mice subjected to angiotensin II infusion. J Mol Cell Cardiol. 2016;100:64-71.

Conway MA, Allis J, Ouwerkerk R, Niioka T, Rajagopalan B, Radda GK. Detection of low phosphocreatine to ATP ratio in failing hypertrophied human myocardium by 31P magnetic resonance spectroscopy. Lancet. 1991;338:973-6.

Davila-Roman VG, Vedala G, Herrero P, de las Fuentes L, Rogers JG, Kelly DP, et al. Altered myocardial fatty acid and glucose metabolism in idiopathic dilated cardiomyopathy. J Am Coll Cardiol. 2002:40:271-7.

Degens $\mathrm{H}$, de Brouwer KF, Gilde AJ, Lindhout M, Willemsen PH, Janssen BJ, et al. Cardiac fatty acid metabolism is preserved in the compensated hypertrophic rat heart. Basic Res Cardiol. 2006;101:17-26.

Dyck JR, Cheng JF, Stanley WC, Barr R, Chandler MP, Brown S, et al. Malonyl coenzyme a decarboxylase inhibition protects the ischemic heart by inhibiting fatty acid oxidation and stimulating glucose oxidation. Circ Res. 2004;94:e78-84

Dyck JR, Hopkins TA, Bonnet S, Michelakis ED, Young ME, Watanabe M, et al. Absence of malonyl coenzyme a decarboxylase in mice increases cardiac glucose oxidation and protects the heart from ischemic injury. Circulation. 2006;114:1721-8.

Folmes CD, Clanachan AS, Lopaschuk GD. Fatty acids attenuate insulin regulation of 5'-AMP-activated protein kinase and insulin cardioprotection after ischemia. Circ Res. 2006;99:61-8.

Gerriets VA, Kishton RJ, Nichols AG, Macintyre AN, Inoue M, Ilkayeva O, et al. Metabolic programming and PDHK1 control CD4+ T cell subsets and inflammation. J Clin Invest. 2015;125:194-207.

Helge JW, Richter EA, Kiens B. Interaction of training and diet on metabolism and endurance during exercise in man. J Physiol. 1996;492(Pt 1):293-306.

Horgan S, Watson C, Glezeva N, Baugh J. Murine models of diastolic dysfunction and heart failure with preserved ejection fraction. J Card Fail. 2014;20:984-95.

Ingwall JS. On substrate selection for ATP synthesis in the failing human myocardium. Am J Physiol Heart Circ Physiol. 2007;293:H3225-6.

Kato T, Niizuma S, Inuzuka Y, Kawashima T, Okuda J, Tamaki Y, et al. Analysis of metabolic remodeling in compensated left ventricular hypertrophy and heart failure. Circ Heart Fail. 2010;3:420-30.

Klotz S, Hay I, Zhang G, Maurer M, Wang J, Burkhoff D. Development of heart failure in chronic hypertensive dahl rats: focus on heart failure with preserved ejection fraction. Hypertension. 2006;47:901-11.

Lai L, Leone TC, Keller MP, Martin OJ, Broman AT, Nigro J, et al. Energy metabolic reprogramming in the hypertrophied and early stage failing heart: a multisystems approach. Circ Heart Fail. 2014;7:1022-31.
Lei B, Lionetti V, Young ME, Chandler MP, d'Agostino C, Kang E, et al. Paradoxical downregulation of the glucose oxidation pathway despite enhanced flux in severe heart failure. J Mol Cell Cardiol. 2004;36:567-76.

Leong HS, Grist M, Parsons H, Wambolt RB, Lopaschuk GD, Brownsey R, et al. Accelerated rates of glycolysis in the hypertrophied heart: are they a methodological artifact? Am J Physiol Endocrinol Metab. 2002;282:E1039-45.

Lewis AJ, Neubauer S, Tyler DJ, Rider OJ. Pyruvate dehydrogenase as a therapeutic target for obesity cardiomyopathy. Expert Opin Ther Targets. 2016:20:755-66.

Liao R, Jain M, Cui L, D'Agostino J, Aiello F, Luptak I, et al. Cardiac-specific overexpression of GLUT1 prevents the development of heart failure attributable to pressure overload in mice. Circulation. 2002;106:2125-31.

Liu B, Clanachan AS, Schulz R, Lopaschuk GD. Cardiac efficiency is improved after ischemia by altering both the source and fate of protons. Circ Res. 1996;79:940-8.

Liu Q, Docherty JC, Rendell JCT, Clanachan AS, Lopaschuk GD. High levels of fatty acids delay the recovery of intracellular $\mathrm{pH}$ and cardiac efficiency in postischemic hearts by inhibiting glucose oxidation. J Am Coll Cardiol. 2002;39: $718-25$.

Lopaschuk GD, Barr R, Thomas PD, Dyck JR. Beneficial effects of trimetazidine in ex vivo working ischemic hearts are due to a stimulation of glucose oxidation secondary to inhibition of long-chain 3-ketoacyl coenzyme a thiolase. Circ Res. 2003;93:e33-7.

Lopaschuk GD, Ussher JR, Folmes CD, Jaswal JS, Stanley WC. Myocardial fatty acid metabolism in health and disease. Physiol Rev. 2010;90:207-58.

Masoud WG, Ussher JR, Wang W, Jaswal JS, Wagg CS, Dyck JR, et al. Failing mouse hearts utilize energy inefficiently and benefit from improved coupling of glycolysis and glucose oxidation. Cardiovasc Res. 2014;101:30-8.

Mori J, Alrob OA, Wagg CS, Harris RA, Lopaschuk GD, Oudit GY. ANG II causes insulin resistance and induces cardiac metabolic switch and inefficiency: a critical role of PDK4. Am J Physiol Heart Circ Physiol. 2013;304:H1103-13.

Morimoto S, Goto T. Role of troponin I isoform switching in determining the $\mathrm{pH}$ sensitivity of $\mathrm{ca}(2+)$ regulation in developing rabbit cardiac muscle. Biochem Biophys Res Commun. 2000;267:912-7.

Nascimben L, Friedrich J, Liao R, Pauletto P, Pessina AC, Ingwall JS. Enalapril treatment increases cardiac performance and energy reserve via the creatine kinase reaction in myocardium of Syrian myopathic hamsters with advanced heart failure. Circulation. 1995;91:1824-33.

Neubauer S. The failing heart-an engine out of fuel. N Engl J Med. 2007:356: $1140-51$.

Neubauer S, Remkes H, Spindler M, Horn M, Wiesmann F, Prestle J, et al. Downregulation of the Na+-Creatine cotransporter in failing human myocardium and in experimental heart failure. Circulation. 1999;100:1847-50.

Nicholl TA, Lopaschuk GD, McNeill JH. Effects of free fatty acids and dichloroacetate on isolated working diabetic rat heart. Am J Phys. 1991;261: H1053-9.

Pereira RO, Wende AR, Olsen C, Soto J, Rawlings T, Zhu Y, et al. GLUT1 deficiency in cardiomyocytes does not accelerate the transition from compensated hypertrophy to heart failure. J Mol Cell Cardiol. 2014;72:95-103.

Piao L, Fang YH, Cadete VJ, Wietholt C, Urboniene D, Toth PT, et al. The inhibition of pyruvate dehydrogenase kinase improves impaired cardiac function and electrical remodeling in two models of right ventricular hypertrophy: resuscitating the hibernating right ventricle. J Mol Med. 2010:88:47-60

Piao L, Sidhu VK, Fang YH, Ryan JJ, Parikh KS, Hong Z, et al. FOXO1-mediated upregulation of pyruvate dehydrogenase kinase-4 (PDK4) decreases glucose oxidation and impairs right ventricular function in pulmonary hypertension: therapeutic benefits of dichloroacetate. J Mol Med. 2013;91:333-46.

Rapp JP, Dene H. Development and characteristics of inbred strains of Dahl saltsensitive and salt-resistant rats. Hypertension. 1985;7:340-9.

Schiaffino S, Gorza L, Ausoni S. Troponin isoform switching in the developing heart and its functional consequences. Trends Cardiovasc Med. 1993;3:12-7.

Schroeder MA, Lau AZ, Chen AP, Gu Y, Nagendran J, Barry J, et al. Hyperpolarized (13)C magnetic resonance reveals early- and late-onset changes to in vivo pyruvate metabolism in the failing heart. Eur J Heart Fail. 2013;15:130-40.

Seymour AM, Giles L, Ball V, Miller JJ, Clarke K, Carr CA, et al. In vivo assessment of cardiac metabolism and function in the abdominal aortic banding model of compensated cardiac hypertrophy. Cardiovasc Res. 2015;106:249-60.

Stanley WC, Morgan EE, Huang H, MCElfresh TA, Sterk JP, Okere IC, et al. MalonylCoA decarboxylase inhibition suppresses fatty acid oxidation and reduces lactate production during demand-induced ischemia. Am J Physiol Heart Circ Physiol. 2005;289:H2304-9. 
Steenbergen C, Deleeuw G, Rich T, Williamson JR. Effects of acidosis and ischemia on contractility and intracellular pH of rat heart. Circ Res. 1977;41:849-58.

Tian R, Nascimben L, Kaddurah-Daouk R, Ingwall JS. Depletion of energy reserve via the Creatine kinase reaction during the evolution of heart failure in Cardiomyopathic hamsters. J Mol Cell Cardiol. 1996;28:755-65.

Ussher JR, Koves TR, Jaswal JS, Zhang L, Ilkayeva O, Dyck JR, et al. Insulinstimulated cardiac glucose oxidation is increased in high-fat diet-induced obese mice lacking malonyl CoA decarboxylase. Diabetes. 2009;58:1766-75.

Ussher JR, Wang W, Gandhi M, Keung W, Samokhvalov V, Oka T, et al. Stimulation of glucose oxidation protects against acute myocardial infarction and reperfusion injury. Cardiovasc Res. 2012;94:359-69.

Vander Heiden MG, Cantley LC, Thompson CB. Understanding the Warburg effect: the metabolic requirements of cell proliferation. Science. 2009;324:1029-33.

Vogel S, Sperelakis N. Blockade of myocardial slow inward current at low pH. Am J Phys. 1977;233:C99-103

Warburg O. On the origin of cancer cells. Science. 1956;123:309-14.

Yamashita T, Honda M, Takatori H, Nishino R, Minato H, Takamura H, et al Activation of lipogenic pathway correlates with cell proliferation and poor prognosis in hepatocellular carcinoma. J Hepatol. 2009;50:100-10.

Zhabyeyev P, Gandhi M, Mori J, Basu R, Kassiri Z, Clanachan A, et al. Pressureoverload-induced heart failure induces a selective reduction in glucose oxidation at physiological afterload. Cardiovasc Res. 2013;97:676-85.

Zhang L, Jaswal JS, Ussher JR, Sankaralingam S, Wagg C, Zaugg M, et al. Cardiac insulin-resistance and decreased mitochondrial energy production precede the development of systolic heart failure after pressure-overload hypertrophy. Circ Heart Fail. 2013:6:1039-48.

Zhong J, Basu R, Guo D, Chow FL, Byrns S, Schuster M, et al. Angiotensinconverting enzyme 2 suppresses pathological hypertrophy, myocardial fibrosis, and cardiac dysfunction. Circulation. 2010;122:717-28.

\section{Submit your next manuscript to BioMed Central and we will help you at every step:}

- We accept pre-submission inquiries

- Our selector tool helps you to find the most relevant journal

- We provide round the clock customer support

- Convenient online submission

- Thorough peer review

- Inclusion in PubMed and all major indexing services

- Maximum visibility for your research

Submit your manuscript at www.biomedcentral.com/submit 